\title{
The incidence of metabolic syndrome in adolescents with different phenotypes of PCOS
}

\author{
Kubra Zengin Altintas, Berna Dilbaz, Derya Akdag Cirik, Runa Ozelci, Tuba Zengin, \\ Osman Nuri Erginay, Serdar Dilbaz \\ Department of Reproductive Endocrinology and Infertility, \\ Etlik Zubeyde Hanım Women's Health Training and Research Hospital, Ankara, Turkey
}

\begin{abstract}
Objectives: To evaluate the incidence of metabolic syndrome in Turkish adolescents with different phenotypes of polycystic ovary syndrome (PCOS).

Material and methods: This cross-sectional study was performed on the Youth Center clinic of a tertiary referral hospital in Turkey. Adolescents with PCOS $(n=144)$ were classified into four phenotype groups according to the presence of oligo/anovulation $(\mathrm{O})$, hyperandrogenism $(\mathrm{H})$, and polycystic ovarian morphology $(\mathrm{P})$ as follows: Phenotype $\mathrm{A}(\mathrm{O}+\mathrm{H}+\mathrm{P})$, Phenotype $B(H+O)$, Phenotype $C(H+P)$, Phenotype $D(O+P)$. The adolescents gave early follicular phase blood samples for endocrine and metabolic tests. The incidence and the presence of parameters of metabolic syndrome were assessed among the four groups.

Results: In total, $54.9 \%$ of the adolescents with PCOS were overweight and $25.7 \%$ had metabolic syndrome. The incidence of metabolic syndrome in Phenotypes A-D were as follows: 39.5\%, 20.5\%, 26.5\%, and 15.2\%, respectively. Although body mass index was higher in the Phenotype A group, insulin resistance was similar in all of the phenotype groups. The most common dyslipidemia was low HDL-C levels and this was present in more than half of the adolescents with PCOS. Both body mass index and total testosterone levels were significantly higher in adolescents with metabolic syndrome in comparison to those without metabolic syndrome.

Conclusions: Although low HDL-C levels and insulin resistance are common PCOS findings in adolescents, the metabolic profile seems to be worse in Phenotype A than the other phenotypes. Therefore, screening programs should evaluate patients based on the known risk factors and phenotypes for adolescents with PCOS.
\end{abstract}

Key words: adolescents, metabolic syndrome, phenotype, polycystic ovary syndrome

Ginekologia Polska 2017; 88, 6: 289-295

\section{INTRODUCTION}

Polycystic ovary syndrome (PCOS) is the most frequent endocrine disorder in reproductive-aged women. Although the exact cause is unknown, current evidence suggests that PCOS is a complex genetic disorder, and excess androgen produced by ovaries during puberty is supposed to be the initiating factor $[1,2]$. PCOS is a clinically and biochemically heterogeneous disease and girls with PCOS are at an increased risk for MS, diabetes mellitus and a decreased cardiovascular event-free survival in advancing age [3]. MS is a combination of multiple risk factors that arise from insulin resistance accompanying abnormal adipose deposi- tion and function. Women with MS are at increased risk for coronary heart disease, diabetes, fatty liver and several cancers. Although this syndrome affects women from infancy to menopause the diagnostic criteria for diagnosis if PCOS in adolescents is still highly controversial as the physiological changes in adolescence and the hormonal inbalance during maturation of the hypotalamo-hypophysis-ovarian axis in adolescents might mimic some symptoms of PCOS [4].

In 2012, PCOS Consensus Workshop group stated that only adolescents that meet the three Rotterdam criteria should be diagnosed with PCOS [5]. According to this description, only phenotype A can be considered as PCOS 
in adolescents. However, there is great heterogeneity in ovarian morphology among adolescents, and the ultrasonographic diagnosis of polycystic appearance of the ovaries before the age of 17 is inaccurate [6]. Moreover, other studies suggested that ovarian hyperandrogenism might not become prominent during early adolescence, and adolescents who had only anovulation and polycystic ovaries might also be diagnosed with PCOS in the follow-up period [7, 8]. Therefore, it is not appropriate to exclude the diagnosis of PCOS in the absence of hyperandrogenism or polycystic ovarian morphology in adolescents. In adults, PCOS is currently diagnosed using the presence of two out of three Rotterdam criteria, and four reproductive phenotypes have been defined. Currently, the issue of the selection of the appropriate criteria for the diagnosis of PCOS in adolescents is still debatable [9]. The American College of Obstetricians and Gynecologists (ACOG) and the Androgen Excess Society (AES) guidelines state that hyperandrogenism is an essential criterion for the diagnosis of PCOS [10-12].

In this study, in order not to overlook the diagnosis of PCOS, we used Rotterdam criteria for diagnosing PCOS in adolescents and analyzed the results for each one of the 4 phenotypes.

\section{OBJECTIVES}

The incidence of MS has been reported as to be as high as $37 \%$ in adolescents with PCOS [13] and AES currently recommends a cardio-metabolic assessment for all adolescents with PCOS [10]. In order to decrease the future cardiovascular mortality, it is crucial to accurately identify the PCOS phenotypes that have and increased risk for MS in adolescents. It is known that the adolescents who do not currently fulfill the three Rotterdam criteria might also be diagnosed as PCOS in the following years, therefore, they might also be a candidate for metabolic syndrome. The hypothesis of the study is Turkish adolescents who met any two of three Rotterdam criteria also has a similar incidence of MS and metabolic risk profile with those who met all three Rotterdam criteria.

\section{MATERIAL AND METHODS Study participants}

This cross-sectional study was conducted at the Youth Center Clinic of Etlik Zubeyde Hanim Women's Health Training and Research Hospital, between January 1, 2014 and December 31, 2014. The hospital's Institutional Review Board approved the study. Written informed consents were obtained from all participants and/or their parents before their participation. Before recruitment to the study, all adolescents were evaluated by a reproductive endocrinology specialist and other etiologies that cause hyperandrogenism were ruled out. The adolescents who were diagnosed to have PCOS were enrolled into the study. A total of 144 consecutive adolescents with newly diagnosed PCOS who had a signed written consent were included. All were at least two years post-menarche during the initial assessment of the study.

The diagnosis of PCOS was based on the presence of two out of three Rotterdam criteria that were proposed in 2003 after exclusion of the other medical conditions that cause hyperandrogenism. Those criteria are: (1) clinical and/or biochemical hyperandrogenism; (2) oligomenorrhea persistent 2 years after menarche, $<6$ menstrual cycles per year, and menstrual cycles intervals $>35$ days; (3) presence of ultrasonographic polycystic ovary (PCO) appearance. Clinical hyperandrogenism is defined as the presence of hirsutism, as determined by a Ferriman Gallwey score of 8 or higher. Presence of acne and alopecia without hirsutism was not enough to fulfill the criterion for clinical hyperandrogenism. Biochemical hyperandrogenism is defined as increased total testosterone ( $>0.73 \mathrm{ng} / \mathrm{mL}$; upper limits of the laboratory). PCO is defined as the presence of at least 12 follicles measuring 2-9 $\mathrm{mm}$, or an ovarian volume greater than $10 \mathrm{~cm}^{3}$. The exclusion criteria were: pregnancy, diabetes mellitus, thyroid disorder, hyperprolactinemia, congenital adrenal hyperplasia or an androgen-secreting tumor, known cardiovascular disease; smoking and/or substance abuse; or drugs use that affect carbohydrate and fat metabolism, such as oral contraceptives and metformin.

During the study period, all adolescents with PCOS were examined by specialists and classified into four phenotypes: Phenotype A: hyperandrogenism $(\mathrm{HA})+$ oligoanovulation $(\mathrm{OA})$ + PCO; Phenotype B: hyperandrogenism + oligoanovulation; Phenotype C: hyperandrogenism + PCO; and Phenotype D: oligoanovulation + PCO.

The demographic characteristics, the medical history and hirsutism score of all the participants were recorded. A complete physical examination for anthropometric measurements was followed by blood pressure measurement.

\section{Study protocol}

All participants underwent anthropometric measurements, such as weight, height, waist, and hip circumference. Body mass index (BMI) was also calculated as the formula of weight divided by the square of height. The participants' blood pressure was also measured. One of the researchers (KZA) performed an ultrasonographic examination (General Electric LOGIQ A5, USA) on all participants to investigate the presence of polycystic ovarian morphology and to exclude other gynecologic pathologies.

Fasting venous blood was taken in the early follicular phase of menstruation and used for biochemical and hormonal analysis. The laboratory investigations included measurement of the lipid profile — triglycerides (TG), cho- 
lesterol, high-density lipoprotein (HDL), low-density lipoprotein (LDL) - glucose metabolism (fasting glucose, fasting insulin) and hormone levels for follicle stimulating hormone (FSH), luteinizing hormone (LH), estradiol (E2), testosterone (T), dehydroepiandrostenedione sulphate (DHEA-S), and 17 hydroxyprogesterone (17-OH-P). Insulin resistance was measured using a homeostasis model assessment score (HOMA-IR) that was calculated as fasting insulin $\times$ fasting glucose/22.5.

Fasting glucose was measured with a Roche module using the glucose oxidase method; fasting insulin was measured with the Siemens Immulite 2500 (Siemens, USA) analyzer using the immune chemiluminescence method. Assays for FSH, LH, E2, DHEA-S, and 17-OH-P were performed using the Roche E170 hormone auto-analyzer (Roche Diagnostics, USA) with electro chemiluminescence. Cholesterol and TG levels were measured using the Roche P800 auto-analyzer (Roche Diagnostics) with the cholesterol esterase and lipoprotein lipase method, respectively. LDL and HDL assays were performed using the Roche P800 auto-analyzer with LDL-cholesterol $2^{\text {nd }}$ generation analysis kit and the HDL-cholesterol $3^{\text {rd }}$ generation analysis kit (Roche Diagnostics).

The International Diabetes Federation (IDF) criteria for MS in children and adolescents was used for the diagnosis of MS [11]. Central obesity, defined as a waist circumference $\geq 80 \mathrm{~cm}$, is the essential component of this definition. The presence of two of the following four criteria was required: (1) TG $\geq 150 \mathrm{mg} / \mathrm{dL}$; (2) $\mathrm{HDL}<50 \mathrm{mg} / \mathrm{dL}$; (3) fasting plasma glucose $\geq 100 \mathrm{mg} / \mathrm{dL}$; and (4) systolic blood pressure $\geq 130 \mathrm{~mm} \mathrm{Hg}$, diastolic blood pressure $\geq 85 \mathrm{~mm} \mathrm{Hg}$.

\section{Statistical analysis}

Data analysis was performed using IBM SPSS Statistics for Windows, Version 21.0. (IBM Corp., Armonk. NY). Compliance of the continuous variables with normal distribution was examined using the Kolmogorov Smirnov test. The descriptive statistics were shown as median values (interquartile range) for the non-normally distributed data and as median \pm standard deviation values for the normally distributed data. The significance of the differences between the mean and median values of the four groups was evaluated using the Student's t test and the Mann Whitney U test, respectively. The nominal variables were analyzed using the Chi-square test. The Mann Whitney $\mathrm{U}$ test was performed in order to test the significance using Bonferroni correction to adjust for multiple comparisons. Unless otherwise specified, $\mathrm{p}<0.05$ is accepted as statistically significant.

\section{RESULTS}

Of the 144 participants, 38 (26.3\%) were Phenotype A, 39 (27.1\%) were Phenotype B, 34 (23.6\%) were Phenotype C, and 33 (22.9\%) were Phenotype D (Tab. 1). The mean age of the entire cohort was $17.25 \pm 1.22$, and the ages of the adolescents in all four phenotypes were similar. Of all adolescents, 110 (76.4\%) had oligo-anovulation while 109 (75.7\%) had hirsutism. The history of PCOS in their family was present in 62 (43.2\%) adolescents. The incidence of family history of PCOS was significantly higher in the Phenotype A group compared to the other phenotypes (63.2\% vs. $35.8 \%$; $\mathrm{p}=0.004$ ) (Tab. 1).

The metabolic and hormonal characteristics of the adolescents with PCOS phenotypes are shown in Table 2. BMI and waist circumference were significantly higher in the Phenotype A group compared to the other phenotypes $(p=0.006$ and $p=0.036$, respectively). Seventy-nine (54.9\%) of the 144 participants in the cohort were overweight. As expected, the Ferriman Gallwey scores, the LH and testosterone levels were significantly higher in the hyperandrogenemic phenotypes (Phenotypes $A, B$, and C) compared to the normoandrogenemic Phenotype $D(p<0,001, p=0,002$ and $\mathrm{p}<0,001$, respectively).

However, the fasting glucose, insulin, and HOMA-IR, were similar in all four of the PCOS phenotypes $(p=0,016$, $p=0,427$ and $p=0,122$, respectively). In addition, no significant differences in the lipid parameters, such as total cholesterol, LDL-C, HDL-C, and TG were observed among the four PCOS phenotypes $(p=0.11, p=0.10, p=0.29$ and $p=0.12$, respectively). The testosterone and LH levels were significantly lower in Phenotype D (OA + PCO) compared to Phenotype A Phenotype B and Phenotype $C(p<0,001$ and

Table 1. Demographic characteristics of the adolescents with 4 phenotypes of PCOS

\begin{tabular}{|l|c|c|c|c|}
\hline & $\begin{array}{c}\text { Phenotype A } \\
\text { HA + AO + PCOS }\end{array}$ & $\begin{array}{c}\text { Phenotype B } \\
\text { HA + AO }\end{array}$ & $\begin{array}{c}\text { Phenotype C } \\
\text { HA + PCO }\end{array}$ & $\begin{array}{c}\text { Phenotype D } \\
\text { AO + PCO }\end{array}$ \\
\hline $\mathrm{N}$ & $38(26.3 \%)$ & $39(27.1 \%)$ & $34(23.6 \%)$ & $33(22.9 \%)$ \\
\hline Age & $17.45 \pm 1.16$ & $17.13 \pm 1.26$ & $17.18 \pm 1.16$ & $17.24 \pm 1.16$ \\
\hline Family history of PCOS & $24(63.2 \%)$ & $15(38.5 \%)$ & $14(41.2 \%)$ & $9(27.3 \%)$ \\
\hline Oligo-anovulation & + & + & - & + \\
\hline Hyperandrogenism & + & + & + & - \\
\hline Polycystic ovaries & + & - & + & + \\
\hline
\end{tabular}


Table 2. Metabolic and hormonal characteristics of the patients with PCOS

\begin{tabular}{|c|c|c|c|c|c|}
\hline Characteristics & Phenotype A & Phenotype B & Phenotype C & Phenotype D & $p$ value \\
\hline BMI & $27.16 \pm 4.14$ & $25.14 \pm 3.87$ & $24.98 \pm 4.05$ & $25.32 \pm 4.14$ & $p=0.006^{a}$ \\
\hline WC $[\mathrm{cm}]$ & $0.84(0.08)$ & $0.77(0.14)$ & $0.78(0.15)$ & $0.79(0.10)$ & $p=0.007^{b}$ \\
\hline Ferrimann-Gallwey score & $10.0(3.0)$ & $9.0(2.0)$ & $9.0(2.0)$ & $3.0(2.0)$ & $\mathrm{p}<0.001^{\mathrm{c}}$ \\
\hline Fasting glucose [mg/dL] & $90(9.0)$ & $87(10.0)$ & $86(9.0)$ & $88(8.0)$ & $p=0.016$ \\
\hline Insulin $[\mu \mathrm{IU} / \mathrm{mL}]$ & $9.98(2.32)$ & $10.20(2.08)$ & $9.76(2.10)$ & $9.15(3.08)$ & $p=0.427$ \\
\hline HOMA-IR & $2.17(0.77)$ & $2.15(0.84)$ & $2.14(0.60)$ & $1.94(0.68)$ & $p=0.122$ \\
\hline Testosterone [ng/mL] & $0.8(0.4)$ & $0.8(0.2)$ & $0.7(0.3)$ & $0.6(0.2)$ & $p<0.001^{d}$ \\
\hline FSH [IU/L] & $5.19(2.12)$ & $5.60(2.12)$ & $5.03(2.59)$ & $5.40(1.87)$ & $p=0.621$ \\
\hline LH [IU/L] & $9.88(2.76)$ & $10.55(2.77)$ & $9.16(4.70)$ & $8.33(2.68)$ & $p=0.002^{e}$ \\
\hline DHEAS $[\mu \mathrm{g} / \mathrm{dL}]$ & $221.0(94.0)$ & $201.5(136.0)$ & $171.5(118.0)$ & $174.5(108.0)$ & $p=0.42$ \\
\hline 17 OH Prog [ng/mL] & $0.98(0.18)$ & $0.96(0.25)$ & $0.95(0.20)$ & $0.89(0.34)$ & $p=0.48$ \\
\hline Cholesterol [mg/dL] & $176.0(42.0)$ & $160(39.0)$ & $182.5(45.0)$ & $154.5(39.0)$ & $p=0.11$ \\
\hline TG [mg/dL] & $134.0(74.0)$ & $95.0(28.0)$ & $100.5(57.0)$ & $100.0(35.0)$ & $p=0.12$ \\
\hline LDL [mg/dL] & $87.5(24.0)$ & $88.0(19.0)$ & $90.0(19.0)$ & $78.0(19.0)$ & $p=0.10$ \\
\hline $\mathrm{HDL}[\mathrm{mg} / \mathrm{dL}]$ & $37.0(9.0)$ & $39.0(10.0)$ & $39.5(11.0)$ & $39.0(7.0)$ & $p=0.29$ \\
\hline
\end{tabular}

Abbreviations: ${ }^{\mathrm{A}} \mathrm{A}$ vs. $B C D,{ }^{b} A$ vs. $B C D,{ }^{c} A B C$ vs. $D,{ }^{d} A B C$ vs. $D,{ }^{e} A B C$ vs. $D$

$\mathrm{BMI}$ - body mass index; WC - waist circumference; HOMA-IR — homeostatic model assessment-insulin resistance; FSH — follicle stimulating hormone; LH — luteinizing hormone; DHEAS — dehydroepiandrosterone sulphate; Prog — Progesteron; TG — triglyceride; LDL — low-density lipoprotein; HDL — high-density lipoprotein

Table 3. The incidence of the parameters of metabolic syndrome in four PCOS phenotypes (N, \%)

\begin{tabular}{|l|c|c|c|c|c|}
\hline & Phenotype A & Phenotype B & Phenotype C & Phenotype D & p value \\
\hline Overweight & $27(71.1 \%)$ & $17(43.6 \%)$ & $17(50.0 \%)$ & $18(54.5 \%)$ & 0.10 \\
\hline Abdominal obesity & $31(81.6 \%)$ & $30(76.9 \%)$ & $27(79.4 \%)$ & $28(24.1 \%)$ & 0.85 \\
\hline Hypertension & $8(21.1 \%)$ & $4(10.3 \%)$ & $5(14.7 \%)$ & $2(6.1 \%)$ & 0.27 \\
\hline $\mathrm{TG} \geq 150[\mathrm{mg} / \mathrm{dL}]$ & $17(44.7 \%)$ & $8(20.5 \%)$ & $8(23.5 \%)$ & $5(15.2 \%)$ & 0.023 \\
\hline $\mathrm{HDL}<50[\mathrm{mg} / \mathrm{dL}]$ & $33(86.9 \%)$ & $31(79.5 \%)$ & $28(82.4 \%)$ & $30(90.9 \%)$ & 0.56 \\
\hline Glu $\geq 100[\mathrm{mg} / \mathrm{dL}]$ & $7(18.4 \%)$ & $2(5.1 \%)$ & $4(11.8 \%)$ & $1(3.0 \%)$ & 0.11 \\
\hline Systolic BP [mm Hg] & $115.0(10.0)$ & $110.0(10.0)$ & $110(10.0)$ & $110(10.0)$ & $p=0.26$ \\
\hline Diastolic BP [mm Hg] & $73.7(10.0)$ & $71.3(5.0)$ & $72.6(10.0)$ & $69.7(5.0)$ & $p=0.19$ \\
\hline Mets (IDF) & $15(39.5 \%)$ & $8(20.5 \%)$ & $9(26.5 \%)$ & $5(15.2 \%)$ & 0.10 \\
\hline
\end{tabular}

Abbreviations: TG — triglyceride; HDL — high-density lipoprotein; Glu — fasting plasma glucose; Mets (IDF) — metabolic syndrome diagnosed with International Diabetes Federation; $\mathrm{BP}$ - blood pressure

$p=0,002$, respectively). The presence of the metabolic syndrome parameters [11] for the four PCOS phenotypes are shown in Table 3. In the entire cohort 37 (25.7\%) adolescents were diagnosed with MS according to IDF criteria. Although it did not reach to a statistical significance, the incidence of MS was highest (39.5\%) in Phenotype A and lowest in Phenotype D (15.2\%).

Out of 144 participants I, seventy-nine (54.9\%) were overweight and 116 (80.5\%) had abdominal obesity. The incidence of being overweight and having abdominal obesity were similar in four phenotypes $(p=0.10$ and $p=0.85$, respectively). Both systolic and diastolic blood pressures were also similar in all phenotypes $(p=0.26$ and $p=0.19$, respectively). High triglyceride levels ( $\mathrm{TG} \geq 150 \mathrm{mg} / \mathrm{dL}$ ) were present in 38 (26.4\%) of all adolescents. Low HDL levels were also present in 122 (84.7\%) of the adolescents and all four phenotypes had similar incidence of low HDL and elevated TG levels.

The endocrinologic and metabolic characteristics of adolescents, with and without MS, were compared in Table 4. The ages of the adolescents with and without MS were similar. However, mean BMI and median waist circumference were significantly higher in the adolescents with MS $(29.63 \pm 3.26$ vs. $24.46 \pm 3.26 ; p<0.001$ and 88 (8) vs. 68 (15); $\mathrm{p}<0.001$, respectively). As expected, the insulin resistance evaluated with HOMA-IR levels were significantly higher in 
Table 4. Comparison of the endocrinologic and metabolic parameters in PCOS women with and without metabolic syndrome ( $\mathrm{n}=144)$

\begin{tabular}{|c|c|c|c|}
\hline Characteristics & $\begin{array}{c}\text { Women } \\
\text { without metabolic syndrome }\end{array}$ & $\begin{array}{c}\text { Women } \\
\text { with metabolic syndrome }\end{array}$ & p value \\
\hline Age (years) & $17.26 \pm 1.22$ & $17.21 \pm 1.25$ & 0.84 \\
\hline BMI $\left[\mathrm{kg} / \mathrm{m}^{2}\right]$ & $24.46 \pm 3.30$ & $29.63 \pm 3.26$ & $<0,001$ \\
\hline$W C[\mathrm{~cm}]$ & $68.0(15.0)$ & $88.0(8.0)$ & $<0,001$ \\
\hline \multicolumn{4}{|l|}{ Metabolic characteristics } \\
\hline Fasting glucose [mg/dL] & $87.0(8.0)$ & $94.0(14.0)$ & $<0,001$ \\
\hline Insulin $[\mu \mathrm{IU} / \mathrm{mL}]$ & $9.2(2.61)$ & $10.8(2.08)$ & $<0,001$ \\
\hline HOMA-IR & $1.98(0.58)$ & $2.56(0.79)$ & $<0,001$ \\
\hline Cholesterol [mg/dL] & $158.0(36.0)$ & $197.0(21.0)$ & $<0,001$ \\
\hline Triglyceride [mg/dL] & $92.0(28.0)$ & $153.0(12.0)$ & $<0,001$ \\
\hline LDL [mg/dL] & $80.0(20.0)$ & $92.5(19.0)$ & 0,003 \\
\hline $\mathrm{HDL}[\mathrm{mg} / \mathrm{dL}]$ & $40.0(10.0)$ & $35.0(9.0)$ & $<0,001$ \\
\hline \multicolumn{4}{|c|}{ Endocrinological characteristics } \\
\hline Testosterone [ng/mL] & $0.70(0.24)$ & $0.82(0.35)$ & 0,004 \\
\hline DHEA-S [ng/mL] & $205.0(135.0)$ & $221.0(117.0)$ & 0.95 \\
\hline FSH [IU/L] & $5.25(2.10)$ & $5.35(2.27)$ & 0.59 \\
\hline LH [IU/L] & $9.37(3.87)$ & $10.11(3.18)$ & 0.21 \\
\hline
\end{tabular}

Abbreviations: BMI — body mass index; WC — waist circumference; HOMA-IR — homeostatic model assessment-insulin resistance; LDL — low-density lipoprotein; $\mathrm{HDL}$ — high-density lipoprotein; DHEA-S — dehydroepiandrosterone sulphate; FSH — follicle stimulating hormone; LH — luteinizing hormone

the MS group compared to those without MS $(2.56(0.79)$ vs. 1.98 (0.58); $p<0.001)$. In concordance with the presence of insulin resistance, the lipid profile results were worse in the adolescents with MS compared to those without MS. The serum triglyceride levels were significantly higher (153 (12) vs. 92 (28); $\mathrm{p}<0.001$ ) and HDL levels were significantly lower (35 (9) vs. 40 (10); p < 0.001) in MS group compared to those without MS. The endocrine profiles were also compared between two groups, and adolescents with MS had higher the serum testosterone levels compared to those without MS (0.82 (0.3) vs. $0.7(0.24) ; p=0,004)$. The DHEA-S levels were similar in both groups (0.95).

\section{DISCUSSION}

Although many previous studies have investigated MS in adult women with PCOS, there is limited data concerning the incidence of MS in adolescents with PCOS [14-16]. In the present study, more than half of the adolescents were overweight and $25.7 \%$ (37/144) were diagnosed with MS using IDF criteria. Although it was not statistically significant, the incidence of MS was highest in the adolescents with Phenotype A (39.5\%) — the phenotype that meets all three Rotterdam criteria - and it was lowest in the normoandrogenemic Phenotype D (15.2\%). Rahmanpour investigated 30 Iranian adolescents with PCOS and reported that 52\% of them were overweight and 33.3\% had MS [14]. Recently, Aydin et al. reported a 7.9\% incidence of MS using Cook criteria in 63 normal-weight Turkish adolescents with PCOS [16].
However, in one large study, the incidence of MS was reported to be as $3.9 \%$ in healthy Turkish adolescent girls [17]. When our study and former studies were taken into consideration, we could conclude that, Turkish adolescents with PCOS have an approximately five-times increased risk for MS compared to healthy adolescents.

Although the pathogenesis of PCOS is unknown, there is an increasing evidence on that insulin resistance has a critical role on the steroidogenic dysregulation of PCOS. Suggesting the role of insulin on ovarian function, patients having severe insulin resistance due to presence of insulin receptor mutations are accompanied by PCOS [18]. In these cases, extreme hyperinsulinemia might stimulate the insulin like growth factor-1 (IGF-1) that results increased androgen production in ovarian thecal cells. Insulin also acts in conjunction with $\mathrm{LH}$ to increase the androgen production in the ovary. According to our results, adolescents with four PCOS phenotypes had similar rates of insulin resistance, as evaluated using HOMA-IR. This finding was in concordance with the results from previous studies [19, 20], and it confirms that insulin resistance is the common underlying feature of PCOS in adolescence.

Although the most probable link between PCOS and MS is insulin resistance and hyperinsulinism, the abdominal obesity also supposed to deteriorate the metabolic dysfunction in women with PCOS [21]. The insulin is a known mediator for the development of obesity. Insulin stimulate the formation of mature adipocytes from preadipocytes by the 
action a common transcription factor named Kruppel-like factor 15 (KLF-15). The probable alteration of KLF-15 expression in girls with PCOS might mediate the obesity, hepatic steatosis and hyperandrogenism. Women with PCOS are also reported to have increased abdominal fat volume and insulin resistance independent from BMI [22]. In the present study, BMI and waist circumference were significantly higher in the Phenotype A group in comparison to the other phenotypes. Therefore, it is not surprising that the incidence of MS was highest in the Phenotype A group. In addition, high TG levels in the Phenotype A group might also be explained by central obesity and increased visceral adiposity. As a component of MS, in our study group, the most common dyslipidemia in adolescents were the low HDL levels ( $\mathrm{HDL}<50$ ). More than half of the patients had low HDL-C levels, and the levels did not differ between the four phenotypes. Fruzetti et al. also confirmed our results in Italian adolescent PCOS patients [19]. In contrast, a multicenter study reported that low HDL-C levels were more common in hyperandrogenemic adult women with PCOS as compared to normoandrogenemic women with PCOS (40.9\% vs. $18.1 \% ; p=0,001$ ) [23]. However, a recent study of 624 postmenopausal women found that endogenous androgen levels did not predict dyslipidemia, whereas HOMA-IR independently predicted HDL-C [24]. As a result, insulin resistance is a common feature of PCOS, and this might explain why all phenotypes had similar HDL levels.

Increased body weight is accepted as a major risk factor for MS. Increased body mass and androgen levels were also known to be related to inflammatory response, and proinflammatory cytokine production like TNF-a also contributes to development of insulin resistance and metabolic syndrome in PCOS [18-25]. In accordance to literature, more than half of the Turkish PCOS adolescents were overweight in the present study. However, there are contradictory results about the prevalence of MS in normal-weight PCOS adolescents. Some authors have proposed that the metabolic profile of normal-weight PCOS adolescents was similar to that of the controls $[26,27]$. In the present study, the incidence of MS in normal-weight Turkish adolescents was $6.1 \%$; that increased to $25.5 \%$ in overweight adolescents and $67.7 \%$ in obese adolescents. As expected, BMI and waist circumference were significantly higher in the PCOS adolescents with MS in comparison to those without MS.

Pathogenesis of metabolic syndrome is primarily related to insulin resistance, systemic inflammation and endothelial dysfunction. Metabolic syndrome and PCOS share common feature of insulin resistance. Recent data favors that intraovarian adrogen excess is the main defect in the majority of PCOS that leads to anovulation, polycystic ovaries and hirsutism. Insulin resistance might also increase androgen production from theca cells in response to $\mathrm{LH}$ and increase adipogenesis. The hyperandrogenemia itself also causes secondary LH elevation and aggravates the steroid dysfunction in PCOS. Therefore, this vicious cycle between insulin and androgen might be speculated to be the underlying cause of metabolic syndrome in PCOS. In the present study, the PCOS adolescents with MS were also found to have higher testosterone levels than those without MS. Similarly, Coviello et al. demonstrated that girls with PCOS had an increased risk of MS associated with increasing concentrations of unbound testosterone levels [13]. Fruzetti et al. showed that PCOS phenotypes with hyperandrogenemia had a more severe lipid profile than those with normal androgen levels [19]. However, in the present study, adolescents with the normoandrogenemic phenotype (Phenotype $D$ ) also had a relatively high (15.2\%) incidence of MS.

The major strength of this study is that, it is one of the few studies that investigated the incidence of MS in four PCOS phenotypes diagnosed with Rotterdam criteria. In addition, the endocrinologic and metabolic profiles of these phenotypes were compared in detail. However, this study also has some limitations. First, because this is a single center study the results cannot be generalized to the entire adolescent population with PCOS. That would require multicenter studies conducted on different ethnic groups. Second, because this is a cross-sectional study, we could not comment on whether or not the insulin resistance or metabolic profile of the adolescent participants changes over time or continues until adulthood. Third, because each group of PCOS phenotypes has relatively small number of patients, we could not make comparison of lean and obese adolescents in each group.

One in four adolescents with PCOS in our population has MS. The incidence of MS is highest among Turkish adolescents with Phenotype $A$, where all of the Rotterdam criteria were fulfilled, and the incidence is lowest in Phenotype $D$, which is the normoandrogenemic phenotype. Regardless of the phenotype, the most common form of dyslipidemia in Turkish adolescents with PCOS is low HDL levels, and one in two has low HDL levels. Obesity and biochemical hyperandrogenism worsen MS in Turkish adolescents with PCOS. In order to evaluate the present risk factors and further development of metabolic syndrome in adolescents with PCOS, follow-up studies should be done.

\section{Conflict of interest}

All authors declare that there is no financial disclosure. 


\section{REFERENCES}

1. Roe AH, Dokras A. The diagnosis of polycystic ovary syndrome in adolescents. Rev Obstet Gynecol. 2011; 4(2): 45-51, indexed in Pubmed: 22102927.

2. Rosenfield RL. Clinical review: Identifying children at risk for polycystic ovary syndrome. J Clin Endocrinol Metab. 2007; 92(3): 787-796, doi: 10.1210/jc.2006-2012, indexed in Pubmed: 17179197.

3. Shaw LJ, Bairey Merz CN, Azziz R, et al. Postmenopausal women with a history of irregular menses and elevated androgen measurements at high risk for worsening cardiovascular event-free survival: results from the National Institutes of Health--National Heart, Lung, and Blood Institute sponsored Women's Ischemia Syndrome Evaluation. J Clin Endocrinol Metab. 2008; 93(4): 1276-1284, doi: 10.1210/jc.2007-0425, indexed in Pubmed: 18182456.

4. Welt CK, Carmina E. Clinical review: Lifecycle of polycystic ovary syndrome (PCOS): from in utero to menopause. J Clin Endocrinol Metab. 2013; 98(12):4629-4638, doi: 10.1210/jc.2013-2375, indexed in Pubmed: 24064685.

5. Fauser BC, Tarlatzis BC, Rebar RW, et al. Consensus on women's health aspects of polycystic ovary syndrome (PCOS): the Amsterdam ESHRE/ /ASRM-Sponsored 3rd PCOS Consensus Workshop Group. Fertil Steril. 2012; 97(1): 28-38.e25, doi: 10.1016/j.fertnstert.2011.09.024, indexed in Pubmed: 22153789.

6. Goodman NF, Cobin RH, Futterweit W, et al. American Association of Clinical Endocrinologists (AACE), American College of Endocrinology (ACE), Androgen Excess and PCOS Society (AES). American Association Of Clinical Endocrinologists, American College Of Endocrinology, And Androgen Excess And Pcos Society Disease State Clinical Review: Guide To The Best Practices In The Evaluation And Treatment Of Polycystic Ovary Syndrome-Part 1. Endocr Pract. 2015; 21(11): 1291-1300, doi: 10.4158/EP15748.DSC, indexed in Pubmed: 26509855.

7. Ibáñez L, de Zegher F, Potau N. Anovulation after precocious pubarche: early markers and time course in adolescence. J Clin Endocrinol Metab. 1999; 84(8): 2691-2695, doi: 10.1210/jcem.84.8.5883, indexed in Pubmed: 10443661

8. Carmina E, Oberfield SE, Lobo RA. The diagnosis of polycystic ovary syndrome in adolescents. Am J Obstet Gynecol. 2010; 203(3): 201.e1-201. e5, doi: 10.1016/j.ajog.2010.03.008, indexed in Pubmed: 20435290.

9. Cırık DA, Dilbaz B. What do we know about metabolic syndrome in adolescents with PCOS? J Turk Ger Gynecol Assoc. 2014; 15(1): 49-55, doi: 10.5152/jtgga.2014.95776, indexed in Pubmed: 24790517.

10. ACOG Committee on Practice Bulletins--Gynecology. ACOG Practice Bulletin No. 108: Polycystic ovary syndrome. Obstet Gynecol. 2009; 114(4): 936-949, doi: 10.1097/AOG.0b013e3181bd12cb, indexed in Pubmed: 19888063.

11. Legro RS, Arslanian SA, Ehrmann DA, et al. Endocrine Society. Diagnosis and treatment of polycystic ovary syndrome: an Endocrine Society clinical practice guideline. J Clin Endocrinol Metab. 2013; 98(12): 4565-4592, doi: 10.1210/jc.2013-2350, indexed in Pubmed: 24151290.

12. Azziz R, Carmina E, Dewailly D, et al. Task Force on the Phenotype of the Polycystic Ovary Syndrome of The Androgen Excess and PCOS Society. The Androgen Excess and PCOS Society criteria for the polycystic ovary syndrome: the complete task force report. Fertil Steril. 2009; 91(2): 456-488, doi: 10.1016/j.fertnstert.2008.06.035, indexed in Pubmed: 18950759.

13. Coviello AD, Legro RS, Dunaif A. Adolescent girls with polycystic ovary syndrome have an increased risk of the metabolic syndrome associated with increasing androgen levels independent of obesity and insulin resistance. J Clin Endocrinol Metab. 2006; 91(2): 492-497, doi: 10.1210/jc.2005-1666, indexed in Pubmed: 16249280.

14. Rahmanpour H, Jamal L, Mousavinasab SN, et al. Association between polycystic ovarian syndrome, overweight, and metabolic syndrome in adolescents. J Pediatr Adolesc Gynecol. 2012; 25(3): 208-212, doi: 10.1016/j.jpag.2012.02.004, indexed in Pubmed: 22578482.

15. Huang J, Ni R, Chen X, et al. Metabolic abnormalities in adolescents with polycystic ovary syndrome in south China. Reprod Biol Endocrinol. 2010 8: 142, doi: 10.1186/1477-7827-8-142, indexed in Pubmed: 21083920.

16. Aydin Y, Hassa H, Burkankulu D, et al. What is the Risk of Metabolic Syndrome in Adolescents with Normal BMI who have Polycystic Ovary Syndrome? J Pediatr Adolesc Gynecol. 2015; 28(4): 271-274, doi: 10.1016/j. jpag.2014.08.011, indexed in Pubmed: 26049937.

17. Agirbasli M, Agaoglu NB, Orak N, et al. Sex hormones and metabolic syndrome in children and adolescents. Metabolism. 2009; 58(9): 1256-1262, doi: 10.1016/j.metabol.2009.03.024, indexed in Pubmed: 19497594.

18. Sen A, Hammes SR. Granulosa cell-specific androgen receptors are critical regulators of ovarian development and function. Mol Endocrinol. 2010; 24(7): 1393-1403, doi: 10.1210/me.2010-0006, indexed in Pubmed: 20501640.

19. Fruzzetti $F$, Perini $D$, Lazzarini $V$, et al. Adolescent girls with polycystic ovary syndrome showing different phenotypes have a different metabolic profile associated with increasing androgen levels. Fertil Steril. 2009; 92(2): 626-634, doi: 10.1016/j.fertnstert.2008.06.004, indexed in Pubmed: 18706545.

20. Bil E, Dilbaz B, Cirik DA, et al. Metabolic syndrome and metabolic risk profile according to polycystic ovary syndrome phenotype. J Obstet Gynaecol Res. 2016; 42(7): 837-843, doi: 10.1111/jog.12985, indexed in Pubmed: 27071345.

21. Fulghesu A, Magnini R, Portoghese E, et al. Obesity-related lipid profile and altered insulin incretion in adolescents with polycystic ovary syndrome. J Adolesc Health. 2010; 46(5): 474-481, doi: 10.1016/j.jadohealth.2009.10.008, indexed in Pubmed: 20413084

22. Du X, Rosenfield RL, Qin K. KLF15 Is a transcriptional regulator of the human 17beta-hydroxysteroid dehydrogenase type 5 gene. A potential link between regulation of testosterone production and fat stores in women. J Clin Endocrinol Metab. 2009; 94(7): 2594-2601, doi: 10.1210/jc.20090139, indexed in Pubmed: 19366843.

23. Daan NMP, Louwers YV, Koster MPH, et al. Cardiovascular and metabolic profiles amongst different polycystic ovary syndrome phenotypes: who is really at risk? Fertil Steril. 2014; 102(5): 1444-1451.e3, doi: 10.1016/j. fertnstert.2014.08.001, indexed in Pubmed: 25239303.

24. Worsley R, Robinson PJ, Bell RJ, et al. Endogenous estrogen and androgen levels are not independent predictors of lipid levels in postmenopausal women. Menopause. 2013; 20(6): 640-645, doi: 10.1097/GME .0b013e318279bd4a, indexed in Pubmed: 23531683.

25. Wu H, Yu Ke, Yang Z. Associations between TNF- $a$ and interleukin gene polymorphisms with polycystic ovary syndrome risk: a systematic review and meta-analysis. J Assist Reprod Genet. 2015; 32(4): 625-634, doi: 10.1007/s10815-015-0449-7, indexed in Pubmed: 25690158.

26. Acién $\mathrm{P}$, Quereda F, Matallín $\mathrm{P}$, et al. Insulin, androgens, and obesity in women with and without polycystic ovary syndrome: a heterogeneous group of disorders. Fertil Steril. 1999; 72(1): 32-40, indexed in Pubmed: 10428145.

27. Cengiz H, Ekin M, Dagdeviren $\mathrm{H}$, et al. Comparison of serum anti-Müllerian hormone levels in normal weight and overweight-obese adolescent patients with polycystic ovary syndrome. Eur J Obstet Gynecol Reprod Biol. 2014; 180: 46-50, doi: 10.1016/j.ejogrb.2014.06.018, indexed in Pubmed: 25036408 